\section{Medizinische Telefonberatung: Die Sicht der FMH}

Die Ergebnisse der nachfolgend präsentierten Studie sind interessant. Aus meiner Sicht sind sie im Ergebnis ein Aufruf an die Marketingverantwortlichen von Versicherern, den Einbau dieses zusätzlichen Angebots in die Police zu prüfen. Dies ist eine Facette des Themas Telefontriage: was hätten die Versicherten gerne?

Welche Rolle kann Telefontriage für die möglichst kosteneffiziente Untersuchung und Behandlung übernehmen? Wenn wir so fragen, wird die Vernetzung zwischen Telefontriagediensten und den übrigen Leistungserbringern entscheidend, wie die übereinstimmenden Kommentare von Andy Fischer und Olivier Kappeler zeigen:

«Die Telemedizin ist eine effiziente Intervention für klar definierte Zielgruppen und Problemstellungen, welche aber nur einen Teil der Bevölkerung betreffen. Eine sorgfältige Abwägung der Indikationen sowie exakte Kenntnisse der Grenzen der Telefontriage sind ausgesprochen wichtig. Telemedizin und die Telefontriage im besonderen müssen sorgfältig in die restliche Struktur des Gesundheitssystems eingebettet werden, um die erwartete Wirksamkeit zu entfalten. Dies bedingt insbesondere eine enge Zusammenarbeit der Telekonsultationszentren mit den Leistungserbringern.» (Dr. med. Andy Fischer, Medgate, Basel).

«Telefontriage ist ein sinnvolles Instrument. Hausarztkontakte beginnen im Notfalldienst oft über einen telefonischen Kontakt. Hier kann eine erste Beurteilung erfolgen; erste Empfehlungen können abgegeben werden; der Zeitpunkt einer Konsultation kann festgelegt, allenfalls auf den nächsten Tag verschoben werden. Wichtig ist die gute Integration in die Behandlungskette. Es ist zu prüfen, ob die Telefontriage einer Region nicht durch die lokale (Haus)ärzteschaft zu betreiben oder mindestens sehr gut zu koordinieren ist.» (Dr. med. Olivier Kappeler, Mitglied FMH-Zentralvorstand, Ressort Managed Care).

Hanspeter Kuhn, Fürsprecher, stv. Generalsekretär FMH

\title{
Nutzung und Akzeptanz von medizinischer Telefonberatung in der Bevölkerung
}

Rolf Schwarz

Korrespondenz: Rolf Schwarz

Director Health Industry ELVIA

Hertistrasse 2

8304 Wallisellen

Tel. 0442833218

Fax 0442833116

rolf.schwarz@elvia.ch

\begin{abstract}
Einleitung
Medizinische Call-Center haben sich in der Schweiz in den letzten fünf Jahren etabliert. Durch Implementierung von medizinischer Telefontriage in die Notfallversorgung kann die Arbeitslast der Grundversorger vermindert werden und Kosten im Gesundheitswesen können gespart werden. In einer Stichprobe von 1007 Personen in der Deutschschweiz nutzen $5 \%$ den Service einer medizinischen Telefonberatung. Ein sinnvolles und gut akzeptiertes Angebot scheint medizinische Telefonberatung für Familien, Personen ohne festen Hausarzt und Patienten mit akuten Beschwerden zu sein. Möglicherweise kommt es auch für Personen mit häufigen Arztbesuchen in Frage. Zentral für die Verbreitung scheint jedoch die Beseitigung des Wissensdefizites der Versicherten um die Existenz eines entsprechenden Angebotes von seiten der Krankenversicherung zu sein.
\end{abstract}

\section{Demand-Management als Ausnahme}

Trotz der Verbreitung von Telemedizinischen Angeboten in der letzten Dekade wird auch heute noch das Telefon in Zusammenhang mit der Gesundheit vor allen für die reine Übermittlung von administrativen, logistischen und medizinischen Information eingesetzt. In den seltensten Fällen werden Behandlungsprozesse heraus aus Arztpraxen oder Behandlungszimmern auf die oft modernste IT-Infrastruktur übertragen. Sicher, Auskünfte für eigene Patienten werden gerne per Telefon oder auch E-Mail gegeben und der Aufwand ist abrechenbar, aber das sogenannte Demand-Mangement durch spezialisierte medizinische Call-Center ist immer noch die Ausnahme statt Regel. Am ehesten wird dies zur Koordination von kritischen medizinischen Abläufen und Krankenrücktransporten im Sinne einer Nothilfe bei unzureichender medizinischer Versorgung im Ausland genutzt.

Medizinische Call-Center haben sich in der Schweiz in den letzten fünf Jahren etabliert. Krankenversicherungen, Einzel- und Gruppenpraxen, ärztliche Notfalldienste und die Pharmaindustrie nehmen deren Dienstleistungen in Anspruch. In Grossbritannien ist die computerunterstützte Telefontriage NHS-Direct seit dem Jahre 2001 landesweit verfügbar. In Dänemark wurden im Zuge der Reform des ambulanten Notfalldienstes medizinische Call-Center etabliert, welche die Verteilung der Patienten auf die jeweiligen Notfallzentren regelt. Im Zuge der Entwicklung einer nationalen eHealth-Infrastruktur wurde dort ein Internet-basierter Personal Health Record etabliert, so dass in der 
Notfallversorgung bereits die medizinische Vorgeschichte des Anrufenden zur Beurteilung herangezogen werden kann.

Eine Übersichtsarbeit hat gezeigt, dass durch Implementierung von medizinischer Telefontriage in die Notfallversorgung die Arbeitslast der Grundversorger vermindert werden kann [1]. Die Notwendigkeit einer Erweiterung der bestehenden Notfallversorgung kann schon bald auch in verschiedenen Regionen der Schweiz gegeben sein, denn zu befürchten ist aufgrund des Nachwuchsmangels bei den Grundversorgern ein Zusammenbruch der Notfallversorgung [2-3].

Neben den qualitativen Aspekten bei der medizinischen Telefonberatung sind die ökonomischen angesichts ständig steigender Gesundheitskosten und Versicherungsprämien nicht zu unterschätzen. So hat das Winterthurer Institut für Gesundheitsökonomie herausgefunden, dass 70\% der Anrufenden ihre Beschwerden anders als medizinische Fachpersonen beurteilen und $63 \%$ ihr ursprünglich beabsichtigtes Hilfesuchverhalten anpassen. Es fand eine Verlagerung vom Notfallbereich in den Bereich Selbstbehandlung und Hausarzt statt und führte somit pro Anruf zu Fr. 50.- unmittelbar eingesparten Kosten [4]. Dies wurde anhand von 2000 Anrufenden errechnet, deren ursprüngliche Handlungsabsicht mit der Handlungsempfehlung sowie der tatsächlich erfolgten Handlung verglichen wurde.

Nach wie vor ist, trotz solcher Erfahrungen in der Etablierung dieser medizinischen Zusatzdienste, die Akzeptanz durch die Versicherten entscheidend, damit diese genutzt werden, wenn Versicherte $\mathrm{zu}$ Patienten werden. Aus diesem Grund wurde von ELVIA eine Befragung zur Nutzung und Akzeptanz medizinischer Telefonberatung in der Bevölkerung beim unabhängigen LINK-Institut in Auftrag gegeben.

\section{Stichprobe}

Im Zeitraum vom 30.1. bis 4.2.2006 wurden durch eine Telefonbefragung von sprachassimilierten Personen in der Deutschschweiz 1007 Interviews mit Personen im Alter von 18 bis 74 Jahren durchgeführt. Die Befragung erfolgte computerunterstützt in einer standardisierten Form. Die Stichprobe war hinsichtlich des Geschlechts gleich verteilt. Zum Zeitpunkt der Befragung waren $24 \%$ zwischen 18-29 Jahre alt, $42 \% 30$ bis 49 Jahre und 34\% 50-74 Jahre alt, d.h. die Stichprobe war annähernd repräsentativ für ein Versichertenkollektiv. Die Mehrheit der Befragten (84\%) waren Schweizer Staatsbürger. Beim Bildungsniveau zeigten sich keine auffälli- gen Verteilungen: Primarschule (5\%), Sekundarschule (13\%), Berufsschule (47\%), Mittelschule (12\%), Seminar/Technikum/HWV (4\%), Universität/ETH/Hochschule (17\%) und keine Angaben machten $2 \%$. Voll erwerbstätig waren $47 \%$, teilweise erwerbstätig 22\% und nicht erwerbstätig 31\%. In einem Single-Haushalt wohnten $22 \%$, eine Haushaltsgrösse von 2 Personen wurde von $29 \%$ berichtet, 3 Personen von $14 \%$, 4 Personen von $23 \%$ und 5 oder mehr Personen von $13 \%$. Das monatliche Einkommen war bei 9\% geringer als Fr. 3000.-, 30\% berichteten Fr. 3000.- bis Fr. 6000.-, $27 \%$ Fr. 6001.- bis Fr. 9000.-, $13 \%$ Fr. 9001.- bis Fr. 12 000.-, mehr als Fr. 12 000.- wurden von $8 \%$ angegeben und $13 \%$ verweigerten oder wussten die Antwort nicht. Bei der Frage, bei welcher Krankenkasse die Teilnehmer grundversichert sind, spiegelten sich die Marktverhältnisse wider.

\section{Verfügbarkeit und Nutzer}

Wie aus Abbildung 1 ersichtlich ist, nutzen nach eigener Auskunft 5\% den Service einer medizinischen Telefonberatung. Weiteren 19\% steht ein solcher Service zur Verfügung, auch wenn dieser bislang nicht genutzt wurde. 14\% der Befragten gaben an, bei ihrer Krankenversicherung kein entsprechendes Angebot zu haben. Der Grossteil von $62 \%$ wusste nicht, ob sie Zugang zu einem solchen Angebot haben oder nicht. Interessanterweise gibt es hier einen Altersunterschied dahingehend, dass $72 \%$ der Befragten, die jünger als 30 Jahre alt sind, nicht wissen, ob dieser Service von ihrer Krankenversicherung angeboten wird und es bei Befragten ab einem Alter von 50 Jahren nur $54 \%$ sind. Dies hängt wahrscheinlich damit zusammen, dass ältere Personen sich prinzipiell mehr mit dem Thema Gesundheit und möglichen Hilfen im Falle einer Erkrankung auseinandersetzen.

Steht eine medizinische Telefonberatung zur Verfügung, dann nutzen diese tendenziell eher Frauen (22\%) als Männer (18\%) und hauptsächlich Personen im Alter von 30-49 Jahren, wo die Nutzungsrate bei $25 \%$ und damit um 7\% höher als in den anderen Altersklassen liegt. In Familienhaushalten, d.h. bei 3 oder mehr Personen im gleichen Haushalt, wird die medizinische Telefonberatung intensiver genutzt (27\%) als in Paar- (19\%) und Single-Haushalten (13\%).

\section{Erkrankung und Nutzung medizinischer Telefonberatung}

Die medizinische Telefonberatung wird häufiger von Personen genutzt, die keinen Hausarzt haben (22\% vs. $13 \%)$. Teilnehmende mit Hausarzt gehen im Durchschnitt seit 13 Jahren zu 
Abbildung 1

Nutzung einer medizinischen Telefonberatung in Prozent von 1007 Befragten aus der Bevölkerung.

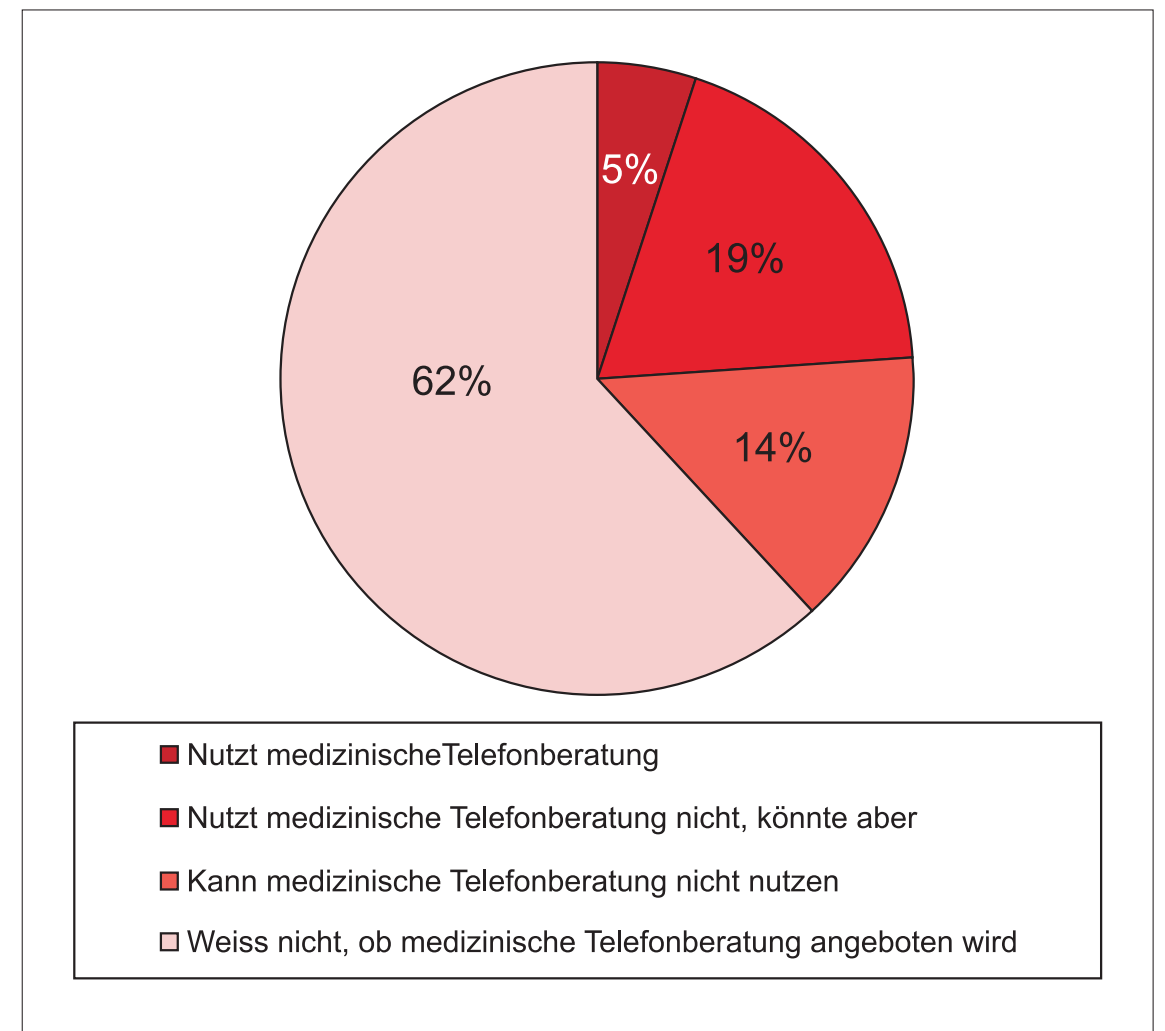

demselben, und, falls dieser nicht verfügbar ist, wenden sich hauptsächlich an die Pikettnummer (39\%), das nächstgelegene Spital (25\%) oder an den Notruf 144 (12\%). Bei den Personen, welche die medizinische Telefonberatung nicht nutzen, obwohl ihnen diese zur Verfügung steht, waren $38 \%$ innerhalb des letzten Jahres nicht beim Arzt vs. $31 \%$ bei den Nutzern einer medizinischen Telefonberatung. Ein signifikanter Unterschied ergibt sich bei Patienten, die innerhalb der letzten 12 Monate ohne Routinekonsultationen häufiger als 5 mal beim Arzt waren, denn in den Gruppen mit der Nutzung bzw. Nutzungsmöglichkeit einer medizinischen Telefonberatung sind dies nur 14\%, während in der Gruppe, der dieses Angebot nicht zur Verfügung steht, dies 19\% sind.

Personen, die eine medizinische Telefonberatung trotz der vorhandenen Möglichkeit nicht in Anspruch nehmen, haben überdurchschnittlich oft ein chronisches Leiden (25\%) und weisen im Schnitt deutlich mehr Arztbesuche auf als Nutzer einer medizinischen Telefonberatung. Über alle Gruppen hinweg wurden als chronische Erkrankungen am häufigsten hoher Blutdruck (13\%), Rückenprobleme (12\%) und Diabetes (10\%) genannt.

\section{Zufriedenheit}

Von den Nutzern einer medizinischen Telefonberatung haben $61 \%$ im vergangenen Jahr den Service zumindest einmal in Anspruch genommen, und der überwiegende Teil der Befragten war mit der Beratung zufrieden (89\%). 44\% der Nutzer würden gegebenenfalls bei geringfügigen gesundheitlichen Problemen eine medizinische Telefonberatung in Anspruch nehmen und 51\% nutzen diese auch, wenn ihr Arzt erreichbar ist. Lediglich $13 \%$ der Nutzer geben an, bereits einmal einen Arzt aufgesucht zu haben, obwohl ihnen im Rahmen einer medizinischen Telefonberatung davon abgeraten wurde.

Befragte, die nach eigener Auskunft Zugang zu einer medizinischen Telefonberatung hatten, begründeten die Nichtnutzung des angebotenen Services in der Regel dadurch, dass es keinen Grund gab, diesen anzurufen (81\%), und nur in $7 \%$ wurde der persönliche Kontakt zum Hausarzt bevorzugt, in $5 \%$ nicht daran gedacht anzurufen wenn es indiziert gewesen wäre, und nur 3\% gaben mangelndes Vertrauen als Grund der Nichtnutzung an. Auf die Frage, «Könnten Sie sich vorstellen, zukünftig den Telefonarzt für medizinische Probleme zu konsultieren, für die Sie früher zum Arzt gegangen wären?», antworteten $59 \%$ mit «Ja». Des weiteren gaben diese Personen an, dass die Auslandsassistance und die medizinische Telefonberatung ihrer Krankenkasse unter der gleichen Telefonnummer zu erreichen sei (23\%), $11 \%$ verneinten dies und $66 \%$ konnten keine Angaben machen. Für die Hälfte dieser $77 \%$ wäre eine einheitliche Telefonnummer allerdings ein wichtiger Service.

\section{Zusammenfassung}

Gegenwärtig ist die medizinische Telefonberatung immer noch ein Nischenangebot im Gesundheitswesen, dessen Potential noch lange nicht ausgeschöpft ist. Die Ergebnisse der Umfrage zeigen, dass es nicht wie vielleicht erwartet die Jungen sind, welche für eine Erweiterung der gegenwärtigen medizinischen Versorgung offen sind, sondern durchaus ältere Personen. Letztere haben, wie bereits erwähnt, naturgemäss ein verstärktes Interesse für Gesundheit und mögliche Hilfen im Falle einer Erkrankung.

Ein sinnvolles Angebot scheint es auch für Familienhaushalte zu sein, da es beispielsweise einfacher ist, einen Telefonhörer in die Hand zu nehmen als Kinder für einen Arztbesuch bereitzumachen oder eine Aufsicht für diese zu organisieren, während man selbst zu einer Konsultation geht. Hat ein Versicherter keinen festen Hausarzt, erscheint es nur logisch, im Falle eines gesundheitlichen Problems eine medizinische 
Telefonberatung in Anspruch zu nehmen. Gleiches gilt im Fall der Nichterreichbarkeit des Hausarztes, denn statt sich gleich an die Notaufnahme des nächstgelegenen Spitals zu wenden, bietet eine medizinische Telefonberatung eine einfache und kostengünstige Alternative. Die erhobenen Daten lassen auch vermuten, dass für Patienten, die häufig zum Arzt gehen, eine medizinische Telefonberatung und -triage eine Alternative bieten würde, und sei es nur darum, sicherzugehen, dass ein anschliessender Arztbesuch wirklich gerechtfertigt ist.

Erstaunlich ist die hohe Zahl der Personen, die nicht wissen, ob dieser Service von ihrer Krankenkasse für sie angeboten wird, denn eigentlich müsste es von zentralem Interesse für die Krankenversicherungen sein, ihre Angebote bei ihren Kunden auch bekanntzumachen.

Grundsätzlich scheint dieser Service eher von Personen mit akuten gesundheitlichen Beschwerden als von chronisch Kranken angenommen zu werden, und diese sind auch bereit, bei geringfügigen gesundheitlichen Problemen anzurufen, selbst wenn ihr Arzt erreichbar ist. Die Compliance, den Empfehlungen einer medizinischen Telefonberatung zu folgen, ist nach eigenen Angaben hoch, wie dies bereits in anderen Publikationen beschrieben wurde [5]. Das Phänomen der Sozialen Erwünschtheit kommt bei diesen Angaben wohl nicht zum Tragen, da diese anonym und einer unabhängigen Institution gegenüber geäussert werden. Abschliessend soll betont werden, dass die Befragten ein grosses Vertrauen und eine Zufriedenheit berichten - ein wichtiger Faktor etwa bei der Auslandsassistance, wo kein bekannter Arzt vorhanden und möglicherweise das Vertrauen in die medizinische Versorgung und Infrastruktur des Gastlandes gering ist.

\section{Literatur}

1 Bunn F, Byrne G, Kendall S. Telephone consultation and triage: effects on the health care use and patient satisfaction. The Cochrane Database of Systematic Reviews. 2004. Volume 4.

2 Grüninger M. Gedanken zum ärztlichen Notfalldienst - eine Zeitbombe? Schweiz Ärztezeitung. 2004;85:2533.

3 Arnet B, von Below G, Zimmermann H. Bei Notfall zuerst den Hausarzt anrufen. Schweiz Ärztezeitung. 2004;85:1052-5.

4 Gatti D, Brügger U, Federspiel B. Gesundheitsökonomische Beurteilung der Telefontriage-Dienstleistung. Winterthurer Institut für Gesundheitsökonomie (WIG); 2005.

5 Meer A, Simonin C, Trapp A, Niemann S, Abel Th. Einfluss der medizinischen computerassistierten Telefontriage auf das Patientenverhalten: erste Erfahrungen aus der Schweiz. Schweiz Ärztezeitung. 2003;84:2160-5.

\section{Schweizerischer eHealthcare-Kongress}

Konferenz \& Fachausstellung, 28./29. September 2006, GZI Forschungszentrum, Nottwil/LU

Der Kongress richtet sich an alle ICT- und Health Professionals der Schweiz und ist Fachmesse und Konferenz zugleich. eHealthCare.ch vereinigt über 1500 führende Health Professionals und ICT-Entscheidungsträger aus dem Spitalsektor, dem ambulanten Sektor, der Industrie, dem Versicherungswesen und der gesamten Gesundheitsbranche.

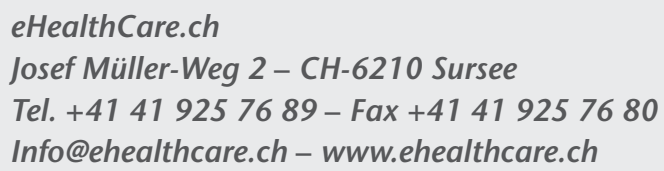

